\title{
ON THE HOMOTOPY TYPE OF THE SPECTRUM REPRESENTING ELLIPTIC COHOMOLOGY
}

\author{
ANDREW BAKER \\ (Communicated by Frederick R. Cohen)
}

\begin{abstract}
In this paper we analyse the homotopy type at primes $p>3$ of the ring spectrum $E \ell \ell$ representing a version of elliptic cohomology whose coefficient ring $E \ell \ell_{*}$ agrees with the ring of modular forms for $S L_{2}(\mathbf{Z})$. For any prime (=maximal) graded ideal $\mathscr{P} \triangleleft E \ell \ell *$ containing the Eisenstein function $E_{p-1}$ as well as $p$, we show that there is a morphism of ring spectra
\end{abstract}

$$
\widehat{E(2)} \longrightarrow(E \ell \ell)_{\mathscr{P}}
$$

and a corresponding splitting

$$
(E \ell \ell)_{\mathscr{P}} \simeq \bigvee_{i} \Sigma^{2 \theta(i)} \widehat{E(2)}
$$

of algebra spectra over $\widehat{E(2)}$ (the $I_{2}$-adic completion of $\left.E(2)\right)$; here ()$\widehat{\mathscr{P}}$ denotes the $\mathscr{P}$-adic completion of the spectrum $E \ell \ell$. Moreover, there is a multiplicative reduction $(E \ell \ell / \mathscr{P})^{*}()$ and we similarly show that there is a splitting of $K(2)$ algebra spectra

$$
E \ell \ell / \mathscr{P} \simeq \bigvee_{i} \Sigma^{2 \theta^{\prime}(i)} K(2)
$$

In each case the indexing $i$ ranges over a finite set.

\section{INTRODUCTION}

Recently, the subject of elliptic cohomology has become the focus of much activity among a wide range of mathematicians and physicists. Although the principal motivation has remained in the interface between quantum field theory, string theory, global analysis on loop spaces and equivariant bordism, it seems likely that the applications to topology itself will also prove fruitful.

The principal attraction of elliptic cohomology in stable homotopy theory is perhaps that it offers the prospect of a geometric interpretation of the notion of $v_{2}$-periodicity, analogous to that of $v_{1}$-periodicity as Bott periodicity

Received by the editors November 10,1988 and, in revised form, January 20,1989.

1980 Mathematics Subject Classification (1985 Revision). Primary 55N22, 14L05, 10 D05.

$K e y$ words and phrases. Elliptic cohomology, $v_{2}$-periodicity, Morava $K$-theory.

The author would like to acknowledge the support of the Science and Engineering Research Council while this work was undertaken. 
in $K$-theory. Unfortunately, to date there is no good geometric description of $E \ell \ell^{*}()$; however, it seems likely that this will appear in the near future.

The purpose of the present paper is to make precise some relationships between $E \ell \ell^{*}()$, Morava $K(2)$-theory and $v_{2}$-periodicity. We use the definition of [Land 1] as modified in [Ba 1], giving a theory whose coefficient ring agrees with the full ring of modular forms for the modular group $S L_{2}(\mathbb{Z})$. We remark that the author's earlier work (see [Ba 1] and [Ba 2]) leads to the conclusion that there is a good set of stable operations available for use in stable homotopy theory and that together with the present results it seems reasonable to look for applications.

Outline. In $\S 1$ we investigate the prime ideals $\mathscr{P}$ of the graded ring $E \ell \ell_{*}$ containing the ideal $\left(p, E_{p-1}\right)$; we show that these are all maximal (i.e. the ring $\left(E \ell \ell_{*}\right)_{(p)}$ has dimension 1) and have the form $(p, \pi)$ where $\pi$ is an irreducible factor of $E_{p-1}$ modulo $p$. We deduce that the residue (graded) field has the form

$$
\mathbf{F}_{p^{d}}\left[Z, Z^{-1}\right]
$$

where $d \geq 1$ and $|Z|=12,8$, or 4 according as to whether $E_{4} \in \mathscr{P}, E_{6} \in \mathscr{P}$ or neither of these holds. We have

$$
\left(E \ell \ell_{*}\right)_{\left(p, E_{p-1}\right)} \cong \prod_{\substack{\bar{\pi} \mid \overline{E_{p-1}} \\(\bar{\pi}) \in \operatorname{Spec}(E \ell \ell * /(p))}}\left(E \ell \ell_{*}\right)_{(p, \pi)}
$$

as topological rings.

In $\S 2$ we use results of J. Igusa and P. Landweber to define formal group laws over the rings $E \ell \ell_{*} / \mathscr{P}$ and $\left(E \ell \ell_{*}\right)_{\mathscr{D}}$ which are strictly isomorphic to the canonical one; then we use results of [BaWü] to produce a further group law on $\left(E \ell \ell_{*}\right)_{\mathscr{P}}$, isomorphic to the canonical one, reducing to the previous one modulo $\mathscr{P}$ and moreover classified by a homomorphism $E(n)_{*} \longrightarrow\left(E \ell \ell_{*}\right)_{\mathscr{P}}$ whose reduction is classified by a homomorphism $K(2)_{*} \longrightarrow E \ell \ell_{*} / \mathscr{P}$.

In $\S 3$ we use results of $\S 1$ to show that there are isomorphisms of rings

$$
\begin{aligned}
& \left(E \ell \ell_{*}\right)_{\mathscr{P}} \cong \bigoplus_{i} \Sigma^{2 \theta(i)} \widehat{E(2)_{*}} \\
& E \ell \ell_{*} / \mathscr{P} \cong \bigoplus_{i} \Sigma^{2 \theta^{\prime}(i)} K(2)_{*}
\end{aligned}
$$

together with corresponding splittings of multiplicative cohomology theories

$$
\begin{aligned}
& \left((E \ell \ell)_{\mathscr{P}}\right)^{*}() \cong \bigoplus_{i} \widehat{E(2)}{ }^{2 \theta(i)+*}() \\
& (E \ell \ell / \mathscr{P})^{*}() \cong \bigoplus_{i} K(2)^{2 \theta^{\prime}(i)+*}() .
\end{aligned}
$$

Associated to the latter are splittings of ring spectra

$$
(E \ell \ell)_{\mathscr{P}} \simeq \bigvee_{i} \Sigma^{2 \theta(i)} \widehat{E(2)}
$$


and

$$
E \ell \ell / \mathscr{P} \simeq \bigvee_{i} \Sigma^{2 \theta^{\prime}(i)} K(2) .
$$

Thus we establish a precise relationship between Morava $K$-theory $K(2)^{*}()$ and elliptic cohomology.

Finally, in the Appendix we discuss the relationship of our results with the theory of supersingular elliptic curves over finite fields.

\section{SUPERSINGULAR REDUCTIONS OF THE RING OF MODULAR FORMS}

Recall from [Ba 1] that there is a multiplicative complex oriented cohomology theory defined on the category of finite $\mathrm{CW}$ complexes $\mathbf{C W} \mathbf{W}^{f}$ by

$$
E \ell \ell^{*}()=E \ell \ell_{*} \otimes_{M U *} M U^{*}()
$$

where we define the graded ring $E \ell \ell_{*}$ by

$$
E \ell \ell_{*}=\mathbb{Z}\left(\frac{1}{6}\right)\left[g_{2}, g_{3}, \Delta^{-1}\right]
$$

with $g_{k} \in E \ell \ell_{4 k}$ and

$$
\Delta=g_{2}^{3}-27 g_{3}^{2} \text {. }
$$

There a is ring homomorphism (genus) $\varphi_{E \ell \ell}: M U_{*} \longrightarrow E \ell \ell_{*}$ constructed using the canonical formal group law $F^{E \ell \ell}$ associated to the elliptic curve

$$
Y^{2}=4 X^{3}-g_{2} X-g_{3}
$$

over $E \ell \ell_{*}$ together with the fact that $M U_{*}$ is identifiable with Lazard's universal ring for formal group laws and possesses a universal group law $F^{M U}$. This is described in detail in [Ba 1] and more generally in [Land 1].

We can identify the group $E \ell \ell_{2 n}$ with the group of weight $n$ modular forms for $S L_{2}(\mathbf{Z})$ which are meromorphic at io and have $q$-expansions with coefficients in $\mathbf{Z}(1 / 6)$ (see [Ba 1]). The corresponding isomorphism of graded rings is effected by the pairings

$$
\left\{\begin{array}{l}
g_{2} \longleftrightarrow \frac{1}{12} E_{4} \\
g_{3} \longleftrightarrow \frac{-1}{216} E_{6}
\end{array}\right.
$$

where $E_{2 n}$ is the weight $2 n$ Eisenstein function, whose $q$-expansion is

$$
E_{2 n}(q)=1-\frac{4 n}{B_{2 n}} \sum_{1 \leq k} \sigma_{2 n-1}(k) q^{k} .
$$

A particular case of importance in the present paper occurs when $p$ is a prime greater than 3 and $2 n=p-1$; then $E_{p-1} \in \mathbf{Z}_{(p)}[[q]]$ is an element of $\left(E \ell \ell_{2(p-1)}\right)_{(p)}($ the localisation at $p)$, and its $q$-expansion satisfies

$$
E_{p-1} \equiv 1 \bmod p
$$


in $\mathbf{Z}_{(p)}[[q]]$.

Notice that $E \ell \ell_{*}$ contains the subring

$$
\mathscr{O}_{*}^{E \ell \ell}=\mathbb{Z}\left(\frac{1}{6}\right)\left[g_{2}, g_{3}\right]
$$

which can be identified with the ring of modular forms holomorphic at io and of course we have

$$
E \ell \ell_{*}=\mathscr{O}_{*}^{E \ell \ell}\left[\Delta^{-1}\right] \text {. }
$$

Convention. From now on we localise at a prime $p \geq 5$, and suppress this from the notation. Thus, in future $E \ell \ell_{*}$ will denote the graded ring $\mathbb{Z}_{(p)}\left[g_{2}, g_{3}, \Delta^{-1}\right]$ and $\mathscr{O}_{*}^{E \ell \ell}$ will denote $\mathbb{Z}_{(p)}\left[g_{2}, g_{3}\right]$. Also, we have $E_{p-1} \in E \ell \ell_{2(p-1)}$. There is a cohomology theory obtained by localising elliptic cohomology at $p$, and this has coefficient ring equal to $E \ell \ell_{*}$; the spectrum representing this localised theory is obtained by $p$-localisation of the spectrum for the version in [Ba 1].

In [Se 3] it is shown that in the ring $\mathscr{O}_{*}^{E \ell \ell} /(p)$, the element $E_{p-1}$ has no repeated irreducible factors (note that $\mathscr{O}_{*}^{E \ell \ell}$ is a unique factorisation domain, as are $\mathscr{O}_{*}^{E \ell \ell} /(p), E \ell \ell_{*}$ and $\left.E \ell \ell_{*} /(p).\right)$ We will be interested in the prime ideals $\mathscr{P} \triangleleft E \ell \ell_{*}$ for which $\left(p, E_{p-1}\right) \subset \mathscr{P}$.

Let $\pi_{1}, \pi_{2}, \ldots, \pi_{d} \in E \ell \ell_{*}$ be a sequence of irreducibles in $E \ell \ell_{*}$ such that

$$
E_{p-1} \equiv \pi_{1} \pi_{2} \cdots \pi_{d} \bmod p .
$$

We can even assume that these all lie in $\mathscr{O}^{E \ell \ell}$ and are irreducible there, since $\Delta$ is a cusp form (i.e. it has no terms in $q$ of degree less than 1) and hence cannot divide $E_{p-1}$. Let $\mathscr{P} \triangleleft E \ell \ell_{*}$ be a prime containing $\left(p, E_{p-1}\right)$; then $\mathscr{P}$ must contain a unique ideal of the form $\left(p, \pi_{k}\right)$. We can say even more, as the following result shows.

Theorem 1.1. Let $\mathscr{P} \triangleleft E \ell \ell_{*}$ be a prime ideal containing $\left(p, E_{p-1}\right)$. Then $\mathscr{P}$ is a maximal graded ideal and the residue ring $E \ell \ell_{*} / \mathscr{P}$ is a finite graded field extension of $F_{p}\left[\Delta, \Delta^{-1}\right]$, having the following form:

(1) $\mathrm{F}_{p}\left[\overline{E_{6}},{\overline{E_{6}}}^{-1}\right]$ if $E_{4} \in \mathscr{P}$;

(2) $\mathrm{F}_{p}\left[\overline{E_{4}},{\overline{E_{4}}}^{-1}\right]$ if $E_{6} \in \mathscr{P}$;

(3) $\mathrm{F}_{p^{d}}\left[W, W^{-1}\right]$ otherwise, where $W \in E \ell \ell_{4}$ satisfies $E_{4} W \equiv E_{6} \bmod p$, and the exponent $d \geq 1$ depends upon $\mathscr{P}$.

In the Appendix we give further details of case (3), using results of [Si] on supersingular elliptic curves over finite fields; in particular, we establish that $d=1$ or 2 .

Proof. We have to consider three distinct cases:

(1) $\overline{\pi_{k}} \sim \overline{E_{4}}$;

(2) $\overline{\pi_{k}} \sim \overline{E_{6}}$;

(3) $\frac{k}{\pi_{k}}$ is not associated to either $\overline{E_{4}}$ or $\overline{E_{6}}$. 
Here all statements refer to elements of the quotient ring $E \ell \ell_{*} /(p)$.

In the cases (1) and (2) we have (using (1.3) and (1.5)) that

$$
E \ell \ell_{*} /\left(p, E_{4}\right)=\mathrm{F}_{p}\left[\overline{E_{6}},{\overline{E_{6}}}^{-1}\right]
$$

and

$$
E \ell \ell_{*} /\left(p, E_{6}\right)=\mathrm{F}_{p}\left[\overline{E_{4}},{\overline{E_{4}}}^{-1}\right]
$$

and so we have the desired result. In case (3) we see that $\pi_{k}$ must be expressible as a homogeneous polynomial in $E_{4}^{3}$ and $E_{6}^{2}$. Hence, for some homogeneous polynomial $f_{k}(X, Y) \in \mathrm{F}_{p}[X, Y]$ we have

$$
\pi_{k} \equiv f_{k}\left(E_{4}^{3}, E_{6}^{2}\right) \bmod p .
$$

Now as $\Delta=1 / 1728\left(E_{4}^{3}-E_{6}^{2}\right)$ does not divide $\pi_{k}$, then $X-Y$ cannot divide $f_{k}(X, Y)$. Hence we can replace $E_{6}^{2}$ by

$$
E_{4}^{3}-1728 \Delta
$$

and obtain

$$
\pi_{k} \equiv \sum_{0 \leq j \leq \operatorname{deg} f_{k}} c_{j} \Delta^{\operatorname{deg} f_{k}-j} E_{4}^{3 j} \bmod p
$$

where $\overline{c_{0}} \neq 0$ in $\mathrm{F}_{p}$. But this means that in the residue ring $E \ell \ell_{*} /\left(p, \pi_{k}\right)$ the element $E_{4}^{3}$ has norm over the subfield $\mathrm{F}_{p}\left[\Delta, \Delta^{-1}\right]$ a nonzero $\mathrm{F}_{p}$ multiple of a power of the unit $\Delta$, hence is itself a unit. A similar argument proves that $E_{6}$ is a unit in the residue ring. By (1.2) we can now deduce that Theorem 1.1 is true, the form of $E \ell \ell_{*} / \mathscr{P}$ in this case following easily since we are reduced to a finite algebraic extension $F_{p^{d}}$ of $F_{p}$ extended by a 4-dimensional periodicity element $W$ as defined above.

Of course, this result determines all the maximal ideals $\mathscr{M} \triangleleft E \ell \ell_{*}$ containing $\left(p, E_{p-1}\right)$. We can use Theorem 1.1 to decompose $E \ell \ell_{*} /\left(p, E_{p-1}\right)$.

Proposition 1.2. There is an isomorphism of rings

$$
E \ell \ell_{*} /\left(p, E_{p-1}\right) \cong \prod_{\substack{\bar{\pi} \mid \overline{E_{p-1}} \\(\bar{\pi}) \in \operatorname{Spec}(E \ell \ell * /(p))}} E \ell \ell_{*} /(p, \pi) .
$$

In turn, this splitting can be lifted to completions.

Proposition 1.3. There is an isomorphism of topological rings

$$
\left(E \ell \ell_{*}\right)_{\left(p, E_{p-1}\right)} \cong \prod_{\substack{\bar{\pi} \mid \overline{E_{p-1}} \\(\bar{\pi}) \in \operatorname{Spec}\left(E \ell \ell_{*} /(p)\right)}}\left(E \ell \ell_{*}\right)_{(p, \pi)} .
$$

2. LUBIN-TATE GROUP LAWS AND SUPER SINGULAR REDUCTIONS OF $E \ell \ell_{*}$

In this section we construct a certain formal group law $F_{\mathscr{P}}$ over each of the completions $\left(E \ell \ell_{*}\right)_{\mathscr{P}}$ where $\mathscr{P}=(p, \pi)$ for $\bar{\pi} \mid \overline{E_{p-1}}$ an irreducible in 
$E \ell \ell_{*} /(p)$. This will turn out to be classified by a homomorphism factoring as

$$
B P_{*} \longrightarrow E(2)_{*} \longrightarrow\left(E \ell \ell_{*}\right)_{\mathscr{D}}
$$

where $E(2)_{*}=v_{2}^{-1} B P_{*} /\left(v_{k}: k \geq 3\right)$. On passing to the residue field $E \ell \ell_{*} / \mathscr{P}$ we find that the reduction of $F_{\mathscr{D}}$ is classified by a homomorphism which factors as

$$
B P_{*} \longrightarrow K(2)_{*} \longrightarrow E \ell \ell_{*} / \mathscr{P}
$$

where $K(2)_{*}=v_{2}^{-1} B P_{*} /\left(v_{k}: 0 \leq k \neq 2\right)$.

We begin with the group law $F^{E \ell \ell}$ over $E \ell \ell_{*}$, which is induced by the genus $\varphi_{E \ell \ell}: M U_{*} \longrightarrow E \ell \ell_{*}$. In [Land 1] it is shown that if we extend $E \ell \ell_{*}$ by adjoining a root $e$ of the cubic

$$
f_{E \ell \ell}(X)=4 X^{3}-g_{2} X-g_{3} \in E \ell \ell_{*}[X]
$$

then the ring $E \ell \ell_{*}(e)$ is isomorphic to

$$
\mathbb{Z}_{(p)}\left[\delta, \varepsilon, \varepsilon^{-1},\left(\delta^{2}-\varepsilon\right)^{-1}\right]
$$

of [Land 1] (recall that we are localised at $p$ ), which is the usual coefficient ring of elliptic cohomology. The standard Euler formal group law $F^{\text {Euler }}$ over $E \ell_{*}(e)$ then becomes strictly isomorphic to $F^{E \ell \ell}$.

If we adjoin 3 distinct roots of $f_{E \ell \ell}$ in some integral domain extension of $E \ell \ell_{*}$, say $E \ell \ell_{*}\left(e_{1}, e_{2}, e_{3}\right)$ where $f_{E \ell \ell}\left(e_{i}\right)=0$, then we have 3 distinct formal group laws $F_{i}^{\text {Euler }}$, each isomorphic to $F^{E \ell \ell}$ by an isomorphism

$$
h_{i}: F^{E \ell \ell} \longrightarrow F_{i}^{\text {Euler }} \text {. }
$$

Now from the work of J. Igusa (see [Ig 1], [Ig 2], and [Land 2]) we have the following result.

Proposition 2.1. For each prime $p \geq 5$ the group law $F^{\text {Euler }}$ over $E \ell \ell_{*}(e)$ has p-series of the form

$$
[p]_{F \text { Euler }}(X) \equiv\left(\frac{-1}{p}\right) \Delta^{\left(p^{2}-1\right) / 12} X^{p^{2}} \bmod \left(p, E_{p-1}\right) .
$$

Proof. This is essentially proved in [Land 2], and needs only the further information that $E_{p-1} \bmod p$ is the Hasse invariant of the elliptic curve of (1.4) (e.g. see $[\mathrm{Si}])$.

Now form the series

$$
h(X)=\frac{1}{3} \sum_{i=1}^{3} h_{i}(X) \in E \ell \ell_{*}[[X]]
$$

which begins with $X$, and so allows us to introduce another formal group law $F$ uniquely specified by the requirement that

$$
h: F^{E \ell \ell} \longrightarrow F
$$


be a strict isomorphism (over $E \ell \ell_{*}$ ). Observe that for any prime $p>3$,

$$
\begin{aligned}
{[p]_{F}(X) } & =\frac{1}{3} \sum_{i=1}^{3} h_{i}\left([p]_{F \text { EU }}(X)\right) \\
& =\frac{1}{3} \sum_{i=1}^{3}[p]_{F_{i}^{\text {Euler }}}\left(h_{i}(X)\right) \\
& \equiv \frac{1}{3}\left(\frac{-1}{p}\right) \Delta^{\left(p^{2}-1\right) / 12} \sum_{i=1}^{3} h_{i}(X)^{p^{2}} \\
& \equiv\left(\frac{-1}{p}\right) \Delta^{\left(p^{2}-1\right) / 12} h(X)^{p^{2}}
\end{aligned}
$$

where the congruences are taken modulo $\left(p, E_{p-1}\right)$.

Now for each such prime $p$, we can construct the canonical $p$-typification $F^{\text {Typ }}$ of $F$ over $E \ell \ell_{*}$ (see [Ad]); this is classified by a ring homomorphism

$$
\varphi_{p}: B P_{*} \longrightarrow E \ell_{*}
$$

satisfying

$$
\varphi_{p}\left(v_{k}\right) \equiv \begin{cases}0, & \text { if } 0 \leq k \neq 2, \\ \left(\frac{-1}{p}\right) \Delta^{\left(p^{2}-1\right) / 12}, & \text { if } k=2,\end{cases}
$$

where $v_{k}$ is the $k$ th Araki generator for $B P_{*}$ (see [Rav]), and once again the congruences are taken modulo $\left(p, E_{p-1}\right)$. But this means that the reduction of $F^{\mathrm{Typ}}$ modulo $\left(p, E_{p-1}\right)$ factors through $K(2)_{*}$, the coefficient ring of the second Morava $K$-theory at the prime $p$.

Now let $\mathscr{P} \triangleleft E \ell \ell_{*}$ be a maximal ideal containing $\left(p, E_{p-1}\right)$. Then upon reducing $\varphi_{p}$ to a homomorphism $K(2)_{*} \longrightarrow E \ell \ell_{*} / \mathscr{P}$ we see that $E \ell \ell_{*}$ becomes a finite graded field extension of

$$
K(2)_{*} \cong \mathrm{F}_{p}\left[\Delta^{\left(p^{2}-1\right) / 12}, \Delta^{-\left(p^{2}-1\right) / 12}\right]
$$

by Theorem 1.1.

By the main results of [BaWü] we can form a new formal group law $F_{\mathscr{P}}$ over $\left(E \ell \ell_{*}\right)_{\mathscr{P}}$, strictly isomorphic to $F^{\mathrm{Typ}}$ by an isomorphism which is the identity modulo $\mathscr{P}$. Moreover, $F_{\mathscr{P}}$ is classified by a homomorphism

$$
\varphi_{\mathscr{P}}: \widehat{E(2)}_{*} \longrightarrow\left(E \ell \ell_{*}\right)_{\mathscr{P}}
$$

covering $K(2)_{*} \longrightarrow E \ell \ell_{*} / \mathscr{P}$ which classifies the above reduction, and where $\widehat{E(2)}$ is the $I_{2}$-adic (Noetherian) completion of $E(2)_{*}$. It is now easy to see that the next result holds.

Proposition 2.2. The topological ring $\left(E \ell \ell_{*}\right)_{\mathscr{D}}$ is a finite rank free algebra over $\widehat{E(2)}$ * identified with $\operatorname{im} \varphi_{\mathscr{D}}$, in a way compatible with the identification of $E \ell \ell_{*} / \mathscr{P}$ as a finite-dimensional $K(2)_{*}$ algebra. 


\section{SUPERSINGULAR REDUCTIONS OF ELLIPTIC COHOMOLOGY AND MORAVA $K$-THEORY}

We are now in a position to state and prove our topological results. For any prime $p>3$ and prime ideal $\mathscr{P} \triangleleft E \ell \ell_{*}$ containing $\left(p, E_{p-1}\right)$, the $\mathscr{P}$ adic completion $\left(E \ell \ell_{*}\right)_{\mathscr{D}}$ is flat over the ring $E \ell \ell_{*}$ since the later is (graded) Noetherian (see [Ma]). Hence, the functor

$$
\left(E \ell \ell_{*}\right)_{\mathscr{P}} \otimes_{E \ell \ell *} E \ell \ell^{*}()
$$

is a cohomology theory on $\mathbf{C} \mathbf{W}^{f}$. The genus $\varphi_{E \ell \ell}$ pushed into $\left(E \ell \ell_{*}\right)_{\mathscr{P}}$ allows us to identify this functor with

$$
\left(E \ell \ell_{*}\right) \hat{\mathscr{P}} \otimes_{M U *} M U^{*}()
$$

and we denote it by

$$
\left(E \ell \ell_{\mathscr{P}}\right)^{*}() \text {. }
$$

By standard arguments, this is easily seen to be a complex oriented multiplicative cohomology theory on $\mathbf{C W}^{f}$, with canonical orientation $x^{E \ell \ell}$ induced by the multiplicative natural transformation

$$
\overline{\varphi_{E \ell \ell}}: M U^{*}() \longrightarrow\left(E \ell \ell_{\mathscr{P}}\right)^{*}()
$$

extending the genus $\varphi_{E \ell \ell}$. The group law associated to this orientation is of course $F^{E \ell \ell}$. Now the group law $F_{\mathscr{P}}$ constructed in $\S 2$ has an isomorphism

$$
h_{\mathscr{P}}: F^{E \ell \ell} \longrightarrow F_{\mathscr{P}}
$$

which provides a new orientation $y^{E \ell \ell}=h_{\mathscr{D}}\left(x^{E \ell \ell}\right)$, induced by a multiplicative natural transformation

$$
\overline{\psi_{\mathscr{P}}}: B P^{*}() \longrightarrow E(2)^{*}() \longrightarrow\left(E \ell \ell_{\mathscr{P}}\right)^{*}() \text {. }
$$

It is easy to verify that using $\overline{\psi_{\mathscr{P}}}$ we have equivalences of functors

$$
\begin{aligned}
\left(E \ell \ell_{\mathscr{P}}\right)^{*}() & \cong\left(E \ell \ell_{*} \hat{)}_{\mathscr{P}} \otimes_{B P_{*}} B P^{*}()\right. \\
& \cong\left(E \ell \ell_{*}\right)_{\mathscr{P}} \otimes_{E(2) *} E(2)^{*}() \\
& \cong\left(E \ell \ell_{*} \hat{)}_{\mathscr{D}} \otimes_{\widehat{E(2)}} \widehat{E(2)}\right)^{*}()
\end{aligned}
$$

where the last theory is defined on $\mathbf{C W}^{f}$ by

$$
\begin{aligned}
\widehat{E(2)}^{*}() & =\widehat{E(2)} \otimes_{E(2) *} E(2)^{*}() \\
& \cong \widehat{E(2)} \otimes_{B P_{*}} B P^{*}()
\end{aligned}
$$

From $\S 2$ we know that $\left(E \ell \ell_{*}\right)_{\bar{\phi}}$ is a finite rank, free $\widehat{E(2)_{*}}$ algebra, and so after choosing a basis, we can naturally identify the theory $\left(E \ell \ell_{\mathscr{P}}\right)^{*}()$ with a finite sum of $\widehat{E(2)}^{*}()$ module theories, via a natural equivalence

$$
\bigoplus_{i} \widehat{E(2)}^{*+2 \theta(i)}() \longrightarrow\left(E \ell \ell_{\mathscr{P}}\right)^{*}()
$$


for some suitable numerical function $\theta$ of the (finite) indexing $i$.

Now this theory (on $\mathbf{C W}^{f}$ ) is given by an inverse limit of the form

$$
\widehat{E(2)}^{*}()=\underset{k}{\lim _{k}}\left(\bigoplus_{i} E(2)^{*}() / I_{2}^{k} E(2)^{*}()\right)
$$

considered in [BaWü] for example. Hence, we have morphisms of representing spectra

$$
\rho: \bigvee_{i} \Sigma^{2 \theta(i)} \widehat{E(2)} \longrightarrow E \ell \ell_{\mathscr{P}}
$$

which by the methods of [BaWü] are unique (up to homotopy) and are morphisms of $\widehat{E(2)}$ module spectra, where the right-hand spectrum inherits a unique module structure from the above multiplicative natural transformation of cohomology theories. But $\rho$ is now easily seen to be an equivalence using Whitehead's Theorem. Thus we have established the following result:

Theorem 3.1. There is an equivalence of $\widehat{E(2)}$ algebra spectra

$$
E \ell \widehat{\ell_{\mathscr{P}}} \simeq \bigvee_{i} \Sigma^{2 \theta(i)} \widehat{E(2)} \text {. }
$$

Upon reduction modulo $\mathscr{P}$ we also have a splitting into copies of $K(2)$. To see this we need to use the version of the Landweber's Exact Functor Theorem valid modulo $I_{n}$ (see [Ya]). Let $\left(M U / I_{2}\right)^{*}()$ be the cohomology theory obtained by killing the regular sequence $p, v_{1}$ in $\left(M U_{*}\right)_{(p)}$, where $v_{1}$ is the first Araki generator in $B P_{*} \subset M U_{*}$. Then there is a cohomology theory

$$
(E \ell \ell / \mathscr{P})^{*}()=E \ell \ell_{*} / \mathscr{P} \otimes_{M U_{*} / I_{2}}\left(M U / I_{2}\right)^{*}()
$$

since the conditions of the Exact Functor Theorem modulo $I_{2}$ hold. Moreover, this is a multiplicative, complex oriented theory and there is a multiplicative natural transformation

$$
\overline{\varphi_{\mathscr{P}}}: P(2)^{*}() \longrightarrow(E \ell \ell / \mathscr{P})^{*}()
$$

where $P(2)^{*}()$ is the theory

$$
P(2)^{*}()=\left(B P_{*} / I_{2}\right) \otimes_{B P_{*} / I_{2}}\left(M U / I_{2}\right)^{*}()
$$

of for instance [Rav]. Of course, $\overline{\varphi_{\mathscr{D}}}$ is obtained using the reduction of $\varphi_{\mathscr{D}}$ modulo $\mathscr{P}$.

Since $\varphi_{\mathscr{P}}: B P_{*} \longrightarrow E \ell \ell_{*} / \mathscr{P}$ factors through $K(2)_{*}$ and

$$
K(2)^{*}() \cong K(2)_{*} \otimes_{P(2) *} P(2)^{*}()
$$

we can deduce that there is a multiplicative natural transformation

$$
\bigoplus_{i} K(2)^{*+2 \theta^{\prime}(i)}() \longrightarrow(E \ell \ell / \mathscr{P})^{*}()
$$

which is an isomorphism (on $\mathbf{C} \mathbf{W}^{f}$ ). Just as before, we have a splitting of module spectra. 
Theorem 3.2. There is an equivalence of $K(2)$ module spectra

$$
\bigvee_{i} \Sigma^{2 \theta^{\prime}(i)} K(2) \longrightarrow E \ell \ell / \mathscr{P} \text {. }
$$

The surprising point about these results is their multiplicative character, since one would generally expect for example $E \ell \ell / \mathscr{P}$ to be a wedge of copies of (suspensions of ) $K(2)$; however, there are many distinct multiplicative structures on wedges of $K(n)$ corresponding to the distinct graded maximal ideals in $v_{n}^{-1} B P_{*}$.

Conjectures. In the above, an essential part was played by the $\mathscr{P}$-adic completions of $E \ell \ell_{*}$. In the case of $K$-theory our techniques yield the fact that the completion of the Adams summand can be identified with $\widehat{E(1)}$; however, it is known that this is possible after only localising at $p$. We conjecture that our results for elliptic cohomology can be sharpened to hold after $\mathscr{P}$-localisation. Were this to be true, we would expect a proof to involve interesting explicit formulae connected with the formal groups which appear. The results modulo $\mathscr{P}$ are clearly best possible.

\section{APPENDIX: SUPERSINGULAR ELLIPTIC CURVES OVER FINITE FIELDS}

We refer the reader to [Si, V§3] for details used in the following discussion.

In accordance with traditional practice, we define the universal $j$-invariant by

$$
j_{E \ell \ell}=E_{4}^{3} \Delta^{-1} \in E \ell \ell_{0} .
$$

Now in Theorem (1-6) case (1), we have $j_{E \ell \ell}=0$ in $E \ell \ell_{*} / \mathscr{P}$, and in case (2) we have $j_{E \ell \ell}=1728$ in $E \ell \ell_{*} / \mathscr{P}$. From [Si] these are seen to correspond to exceptional values of the $j$-invariant. On the other hand, in case (3) we have that the irreducible element

$$
f_{k}^{\prime}\left(E_{4}^{3}, \Delta\right)=\sum_{0 \leq j \leq \operatorname{deg} f_{k}} c_{j} \Delta^{\operatorname{deg} f_{k}-j} E_{4}^{3 j} \in \mathbb{F}_{p}\left[E_{4}^{3}, \Delta\right]
$$

is associated to an irreducible

$$
f_{k}^{\prime \prime}\left(j_{E \ell \ell}\right)=f_{k}^{\prime}\left(E_{4}^{3} \Delta^{-1}, 1\right)
$$

which has $j_{E \ell \ell}$ as a zero in $E \ell \ell_{*} / \mathscr{P}$.

Now recalling that in this situation we have $E \ell \ell_{*} / \mathscr{P}=\mathbb{F}_{p}\left[W, W^{-1}\right]$, we can consider the elliptic curve

$$
\mathbf{E l l}_{0}: Y^{2}=4 X^{3}-\gamma_{2} X-\gamma_{3}
$$

defined over $E \ell \ell_{0}$, where $\gamma_{2}=1 / 12 E_{4} W^{-2}$ and $\gamma_{3}=-1 / 216 E_{6} W^{-3}$. As $E \ell \ell_{0}$ is a finite field, we are now in the situation dealt with in [Si, V§3] and moreover, the $j$-invariant of this curve is $j_{E / \ell}$. Silverman's results include the fact that $j_{E \ell\}} \in \mathbb{F}_{p^{2}}$ and hence we see that the polynomial $f_{k}^{\prime \prime}\left(j_{E \ell \ell}\right)$ is of degree 1 or 2 . From this, we deduce that $f_{k}^{\prime}$ is homogeneous of degree 1 or 2 . 
Now using the identity (1.3) together with the definition of $W$, we obtain

$$
E_{4} \equiv \frac{j_{E \ell \ell} W^{2}}{\left(j_{E \ell \ell}-1728\right)} \quad \bmod \mathscr{P}
$$

and

$$
E_{6} \equiv \frac{j_{E \ell \ell} W^{3}}{\left(j_{E \ell \ell}-1728\right)} \quad \bmod \mathscr{P}
$$

implying that both $E_{4}$ and $E_{6}$ give elements of $\mathbb{F}_{p^{2}}\left[W, W^{-1}\right]$, and even $\mathbb{F}_{p}\left[W, W^{-1}\right]$ if $j_{E \ell \ell}$ is itself of degree 1 over $\mathbb{F}_{p}$. The rank of $E \ell \ell_{*} / \mathscr{P}$ over $K(2)_{*}$ is of course $\left(p^{2}-1\right) d / 2$.

Finally, we note that the number of nonisomorphic supersingular elliptic curves over the algebraic closure of $\mathbb{F}_{p}$ is determined in [Si] as a function of $p$.

\section{ACKNOWLEDGMENTS}

I would like to thank the following for arousing my interest in elliptic cohomology and providing interesting discussions and encouragement: Robin Chapman, Francis Clarke, Peter Landweber, Haynes Miller, Serge Ochanine, Nigel Ray, and Bob Stong.

\section{REFERENCES}

[Ad] J. F. Adams, Stable homotopy and generalised homology, University of Chicago Press, Chicago.

[Ba 1] A. Baker, Hecke operators as operations in elliptic cohomology, preprint, 1988.

[Ba 2] _ Elliptic cohomology, p-adic modular forms and Atkin's operator $\mathrm{U}_{p}$, preprint, 1988.

[BaWü] A. Baker and U. Würgler, Liftings of formal group laws and the Artinian completion of $v_{n}^{-1} B P$, preprint, 1988 .

[Ig 1] J. Igusa, On the transformation theory of elliptic functions, Amer. J. Math. 81 (1959), 436-52.

[Ig 2] On the algebraic theory of elliptic modular functions, J. Math. Soc. Japan 20 (1968), 96-106.

[Ka] N. Katz, p-adic properties of modular schemes and modular forms, Lecture Notes in Mathematics 350 (1973), 69-190.

[Ko] N. Koblitz, Introduction to elliptic curves and modular forms, Springer-Verlag, New York.

[Land 1] P. S. Landweber, Elliptic cohomology and modular forms, Lecture Notes in Mathematics 1326 (1988), 55-68.

[Land 2] _ Supersingular elliptic curves and congruences for Legendre polynomials, Lecture Notes in Mathematics 1326 (1988), 69-93.

[Ma] H. Matsumura, Commutative ring theory, Cambridge University Press, Cambridge.

[Rav] D. C. Ravenel, Complex cobordism and the stable homotopy Groups of spheres, Academic Press, London. 
[Se 1] J.-P. Serre, Cours d'arithmétique, Presses Universitaires de France, Vendôme.

[Se 2] _ Formes modulaires et fonctions zeta p-adiques, Lecture Notes in Mathematics 350 (1973), 191-268.

[Se 3] _ Congruences et formes modulaires, Séminaire Bourbaki, Vol 24 ${ }^{\mathrm{e}}$, no. 416, Lecture Notes in Mathematics 317 (1971/2), 319-38.

[Si] J. Silverman, The arithmetic of elliptic curves, Springer-Verlag, New York.

[Ya] N. Yagita, The exact functor theorem for $B P_{*} / I_{n}$, Proc. Jap. Acad. 52 (1976), 1-3.

Department of Mathematics, Manchester University, Manchester, M13 9PL, ENGLAND 